\title{
Перераспределение токов и тепловых потерь в реверсивно-включаемом динисторе при наличии поперечного перепада температуры
}

\author{
(C) А.В. Горбатюк ${ }^{1}$, Б.В. Иванов ${ }^{2}$ \\ ${ }^{1}$ Физико-технический институт им. А.Ф. Иофрфе РАН, Санкт-Петербург, Россия \\ ${ }^{2}$ Санкт-Петербургский государственный электротехнический университет „ЛЭТИ“, Санкт-Петербург, Россия \\ E-mail: agor.pulse@mail.ioffe.ru
}

Поступило в Редакцию 1 октября 2018 г.

Исследованы эффекты поперечного перепада температуры в реверсивно-включаемых динисторах, учет которых важен для высоковольтных частотных динисторных сборок из-за проблем распределенного охлаждения конструкции. Дано имитационное описание пространственной динамики зарядов и тепла в динисторных чипах при коммутации повторяющихся импульсов тока. Предложен оценочный метод расчета максимальной частоты повторения, согласованной с мощностью теплоотвода.

DOI: 10.21883/PJTF.2019.01.47160.17547

Кремниевые реверсивно-включаемые динисторы (РВД) [1-5] в последние годы начинают применяться в высоковольтных частотных генераторах субмикросекундных импульсов [6,7]. Перспективной основой для этого могут послужить новые модификации РВД с оптимизированной структурой и экономичным способом реверсивной накачки $[8,9]$, численное моделирование которых обнаруживает более высокие показатели, чем для описанных в [6,7] прототипов. Так, предлагаемые варианты с площадью чипа $\sim 1 \mathrm{~cm}^{2}$ и рабочим напряжением $U \sim 2.5-5.0 \mathrm{kV}$ в идеализированных условиях с однородной по площади температурой $T \sim 400 \mathrm{~K}$ смогли бы передавать в нагрузку энергию $\sim 0.25-2.0 \mathrm{~J}$ импульсами длительностью 250-500 ns при энергии потерь $\sim 20-200 \mathrm{~mJ}$ за импульс. Далее вопрос о допустимых частотах непрерывной генерации таких импульсов $f_{m}$ обсуждается с учетом специфических особенностей высоковольтных $(\geq 100 \mathrm{kV})$ генераторов.

В качестве коммутирующих узлов таких генераторов используются последовательные сборки из большого числа переключающих полупроводниковых чипов, перемежающихся с теплопроводящими пластинами для поперечного выноса тепла из объема во внешний радиатор (см. пример на рис. 8 в работе [10]). Поэтому в каждом чипе по поперечному направлению должно наблюдаться определенное снижение температуры, что из-за сильных температурных зависимостей инжекции может заметно сказаться на распределенных по площади степени модуляции проводимости, плотности тока и энергии джоулевых потерь и в итоге существенно повлиять на диапазон допустимых частот генерации.

Для частотных РВД количественный анализ неоднородных взаимосвязанных электротепловых эффектов ранее не проводился, поэтому именно он составляет цель настоящей работы.

Будем считать, что все последовательные звенья коммутирующей сборки находятся в одинаковых физиче- ских условиях. Типичная геометрия единичного звена имеет форму цилиндра с радиусом $r_{m}$, который состоит из находящихся в хорошем электротепловом контакте пластин переключающего чипа РВД $(\mathbf{S})$ и теплоотвода $(\mathbf{T})$ толщиной $w$ и $h$ каждая (см. вставку на рис. 1). Механизм функционирования такого звена представляется более сложным, чем для случаев из работ $[8,9]$, из-за появления новых, трудно сопоставимых пространственных и временны́х масштабов. Радиус звена сильно превосходит по размеру все другие параметры реальной геометрии, включая размеры дискретных элементов ячеистой интегральной структуры РВД $r_{m} \gg \Sigma w_{k}+h>d_{0}$, где $w_{k}$ - толщины отдельных слоев вертикального $P N P N$-профиля легирования, $d_{0}-$ расстояние между шунтами эмиттеров [8,9]. Поэтому перепады температуры по радиусу должны многократно превышать перепады по вертикальному измерению: $\delta T(r) \gg \delta T(x) \sim 0$. Что касается временны́х масштабов, то они диктуются как физикой РВД, так и техническими особенностями частотно-импульсного режима. Времена многостадийного развития инжекции и тепловыделения в слое за один импульс составляют $10^{-9}-10^{-6} \mathrm{~s}$. Время вертикального перераспределения тепла по $x$ между слоями $\mathbf{S}$ и $\mathbf{T}$ задается периодом цикла $10^{-4}-10^{-3} \mathrm{~s}$. А распределенный по $r$ съем тепла, циклически пополняемого в Т-слое, и его поперечный вывод в радиатор, сопровождающийся временны́м усреднением и установлением квазистатического профиля $T_{\text {stat }}(r)$, затягивается до $10^{-1} \mathrm{~s}$.

Получить полное самосогласованное описание этой задачи практически нереально из-за необходимости чрезвычайно плотной дискретизации расчетных сеток. С целью нахождения замкнутого ее описания в физически прозрачных терминах мы прибегаем к ряду упрощений. Разделив геометрию единичного звена по радиусу на две параллельные части $0<r<r^{\prime}$ и $r^{\prime}<r<r^{\prime \prime}=r_{m}$, получим дискретное ее разбиение на 


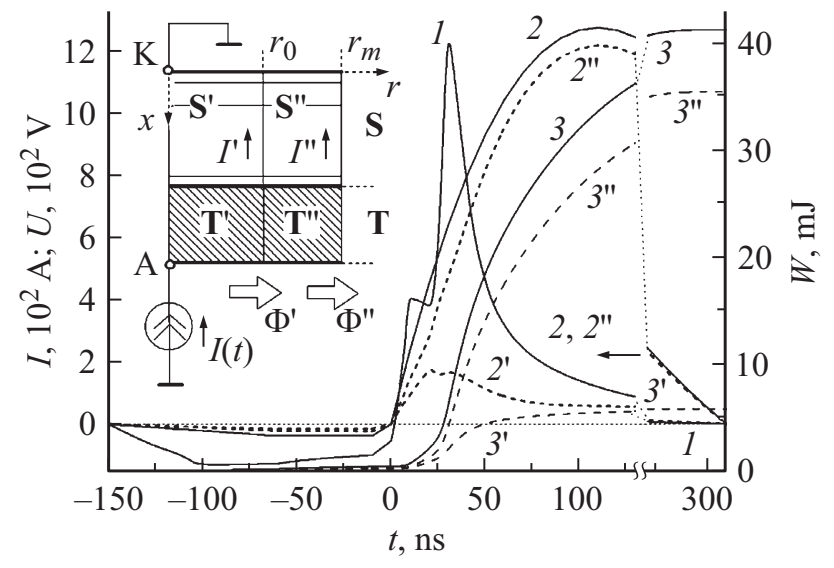

Рис. 1. Переходные характеристики чипа РВД и его составных частей (пояснение в тексте). На вставке - функциональная электротепловая блок-схема повторяющегося звена высоковольтной сборки РВД.

центральный и периферийный сегменты чипа $\left(\mathbf{S}^{\prime}\right.$ и $\left.\mathbf{S}^{\prime \prime}\right)$ и примыкающие к ним участки теплоотвода $\left(\mathbf{T}^{\prime}\right.$ и $\left.\mathbf{T}^{\prime \prime}\right)$. Ввиду плотного размещения большого числа $\left(\sim 10^{4}\right.$ единиц) маленьких шунтированных ячеек в типовых РВД (подробнее см. в [8,9]), а также существенного ослабления влияния точечных шунтов при больших токах считается, что в каждом из сегментов $\mathbf{S}^{\prime}$ и $\mathbf{S}^{\prime \prime}$, дискретных по $r$, инжекция одномерна по $x$. Радиальные потоки тепла $\Phi^{\prime}$ и $\Phi^{\prime \prime}$ через границы сегментов $\mathbf{T}^{\prime}$ и $\mathbf{T}^{\prime \prime}$ обозначены стрелками на рис. 1. Интегральная величина потока $\Phi^{\prime \prime}$ через цилиндрическую поверхность внешней границы $\mathbf{T}^{\prime \prime}$ задает требуемую для охлаждения одного звена мощность внешнего теплоотвода. Далее реальное распределение $T(t, r)$, связанное с $\Phi^{\prime \prime}$, будет аппроксимировано прямоугольной ступенькой с усредненными по периоду $1 / f_{m}$ и по объемам сегментов $\mathbf{S}^{\prime}$ и $\mathbf{S}^{\prime \prime}$ значениями температуры с перепадом $\delta T=T^{\prime}-T^{\prime \prime}>0$. Процедура согласования задаваемых величин $\delta T\left(T^{\prime}, T^{\prime \prime}\right)$ и интенсивности охлаждения описана далее при расчете допустимых частот генерации.

В качестве опорного для анализа мы выбрали пример РВД-1 из работы [8]. Эффективная рабочая площадь всего чипа, как и в [8], была принята равной $A=1 \mathrm{~cm}^{2}$, а площади сегментов для простоты были заданы одинаковыми и равными $A^{\prime}=A^{\prime \prime}=A / 2$.

Численное описание динамики распределенных по $x$ потенциалов, электрического поля, концентраций электронов и дырок, а также создаваемых ими потоков зарядов в сегментах $\mathbf{S}^{\prime}$ и $\mathbf{S}^{\prime \prime}$, подчиняющихся самосогласованной системе уравнения Пуассона и нестационарных уравнений непрерывности токов носителей, проводилось на основе имитационного программного продукта TCAD SENTAURUS [11].

Форма импульса тока через контакты А и К задавалась близкой к рассчитанной в [9]. Для стадии $-t_{R}<t<0$, где $t_{R}-$ время накачки, она трапецеидальная (рис. 1). Ее параметры уточняют- ся далее в конкретных примерах с пробными значениями заряда реверсивного накачки $Q_{R}=\int|I(t)| d t$. Для стадии коммутации $t>0$ использовалась формула $I(t)=I_{0} \exp \left(-t / t_{r e l}\right) \sin \left(2 t / t_{F}\right)$ с коэффициентами $I_{0}=2.46 \mathrm{kA}, t_{r e l}=200 \mathrm{~ns}$ и $t_{F}=310 \mathrm{~ns}$.

На рис. 1 представлены результаты имитационных расчетов динамических переменных задачи для случая с профилем легирования катодного транзистора, показанным на рис. 2, с температурами $T^{\prime}=420 \mathrm{~K}$ и $T^{\prime \prime}=380 \mathrm{~K}$ и зарядом реверсивной накачки $Q_{R}=3.75 \mu \mathrm{C}$. Сплошными кривыми 1-3 на рис. 1 обозначены соответственно временны́е зависимости падения напряжения $U(t)$ между контактами А и $\mathrm{K}$, полного тока $I(t)$ и текущего значения интеграла джоулевых потерь $W(t)=\int I U d t$. Текущие значения частей этих переменных $I^{\prime}, W^{\prime}$ и $I^{\prime \prime}, W^{\prime \prime}$ в центральном $\mathbf{S}^{\prime}$ и периферийном $\mathbf{S}^{\prime \prime}$ сегментах обозначены кривыми $2^{\prime}, 3^{\prime}$ и $2^{\prime \prime}, 3^{\prime \prime}$.

Как и ожидалось, наблюдается опережающий рост текущих значений $I^{\prime \prime}(t)$ над $I^{\prime}(t)$. Различие между ними начиная с 1.5 раз на стадии накачки быстро усиливается на стадии обострения выброса напряжения на кривой 1 и доходит до 20 раз к моменту максимума рабочего импульса $I(t)$. Практически весь ток за это время вытесняется из центральной части $\mathbf{S}^{\prime}$ в периферийный сегмент $\mathbf{S}^{\prime \prime}$, а его плотность удваивается. Переходный пик напряжения при $t \sim 30 \mathrm{~ns}$ достигает максимума $U_{m}=1.2 \mathrm{kV}$, что уже соизмеримо с рабочим напряжением для прототипа из работы [8] и свидетельствует о перегрузке по току. Именно в интервале времен 20-40ns, где одновременно велики и $U(t)$, и $I(t)$, происходит быстрый прирост интегралов потерь, разный по темпу в различных сегментах. В результате к концу импульса интегральные потери в сегменте $\mathbf{S}^{\prime}$ оказываются почти в 8 раз меньшими, чем в сегменте $\mathbf{S}^{\prime \prime}$.

Отмеченные особенности временны́х разверток $U(t)$, $I(t)$ и $W(t)$ могут быть объяснены на основе анализа

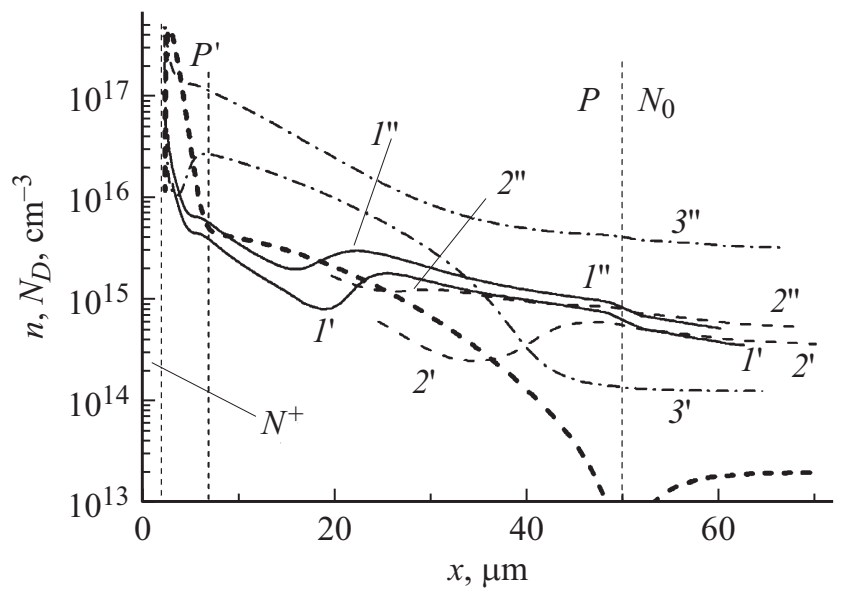

Рис. 2. Распределения концентраций электронов $n$ в катодной окрестности центрального $\mathbf{S}^{\prime}$ и периферийного $\mathbf{S}^{\prime \prime}$ сегментов РВД в различные моменты времени $t_{3}>t_{2}>t_{1}>0$. Жирная штриховая линия $\left(N^{+} P^{\prime} P N_{0}\right)$ - часть профиля концентраций легирования $N_{D}$. Остальные обозначения поясняются в тексте. 


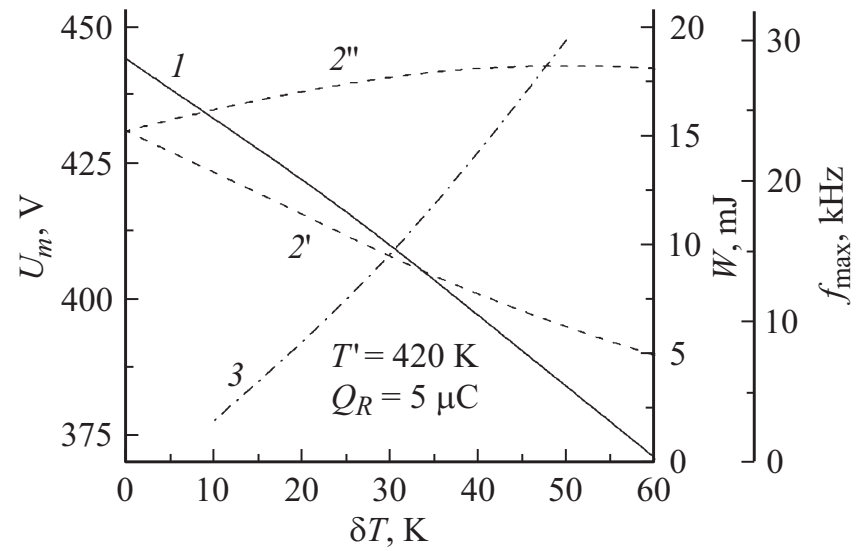

Рис. 3. Зависимости от перепада температуры $\delta T=T^{\prime}-T^{\prime \prime}$ величин пикового напряжения $U_{m}$ на чипе $(1)$ и джоулевых потерь энергии за импульс $W^{\prime}$ и $W^{\prime \prime}\left(2^{\prime}, 2^{\prime \prime}\right)$ в сегментах $\mathbf{S}^{\prime}$ и $\mathbf{S}^{\prime \prime}$, а также максимально допустимой частоты генератора $f_{\max }$ (3) при параметрах теплоотвода $h=0.1 \mathrm{~cm} \mathrm{и}$ $\kappa=\kappa_{\mathrm{Cu}}=3.9 \mathrm{~W} \cdot \mathrm{cm}^{-1} \cdot \mathrm{K}^{-1}$ (медь).

профилей распределений инжектированных электронов в двух выделенных сегментах чипа. На рис. 2 эти профили показаны для моментов $t_{1}=16 \mathrm{~ns}, t_{2}=20 \mathrm{~ns}$ и $t_{3}=110 \mathrm{~ns}$ кривыми $l^{\prime}-3^{\prime}$ и $l^{\prime \prime}-3^{\prime \prime}$, соответствующими начальной стадии формирования фронта включения, переходу к резкому обострению импульса $U(t)$ и прохождению максимума импульса тока $I(t)$ на рис. 1. Видно, как к моменту $t_{1}$ в $P$-базе катодного транзистора уже появляются участки концентрационного обеднения, расширяющиеся к моменту $t_{2}$ к аноду за счет оттеснения накопленной там плазмы при едва начинающемся инжекционном пополнении электронов из катода (происходит трансформация форм $1^{\prime} \rightarrow 2^{\prime}$ и $1^{\prime \prime} \rightarrow 2^{\prime \prime}$ ). При этом изза более сильного температурного подавления инжекции в сегменте $\mathbf{S}^{\prime}$ его $P$-база истощается раньше, а начинает пополняться позже, чем в сегменте $\mathbf{S}^{\prime \prime}$.

Для $t>t_{2}$ характер трансформаций электронных профилей в сегментах $\mathbf{S}^{\prime}$ и $\mathbf{S}^{\prime \prime}$ резко меняется. Если в периферийной части происходит усиление инжекционной модуляции по всему объему сегмента (переход $\left.2^{\prime \prime} \rightarrow 3^{\prime \prime}\right)$, то в сегменте $\mathbf{S}^{\prime}$ область пониженной концентрации проникает в $N_{0}$-базу при ограниченном пополнении электронами катодной окрестности $P$-базы $\left(\right.$ переход $\left.2^{\prime} \rightarrow 3^{\prime}\right)$. Заметим, что к моменту $t_{3}$ минимальные концентрации электронов в сегменте $\mathbf{S}^{\prime \prime}$ не поднимаются выше $(2-5) \cdot 10^{15} \mathrm{~cm}^{-3}$, что на порядок ниже, чем в микросекундных РВД. Это свидетельствует о незавершенности процесса концентрационной модуляции прибора в иллюстрируемом случае.

Для количественного описания зависимостей электротепловых эффектов от перепада $\delta T=T^{\prime}-T^{\prime \prime}$ в оптимизированных модификациях РВД было выполнено их имитационное компьютерное моделирование для 15 точек из интервала изменения $\{\Delta T\}=\{0,60 \mathrm{~K}\}$ при откорректированных значениях некоторых параметров.
Величина заряда накачки к моменту $t=0$ составляла $Q_{R}=5 \mu \mathrm{C}$. Поверхностная концентрация профиля легирования $P$-базы РВД равнялась $N_{p}^{s}=1 \cdot 10^{15} \mathrm{~cm}^{-3}$. Температура сегмента $T^{\prime}=420 \mathrm{~K}$ полагалась фиксированной и примерно равной максимально допустимой рабочей температуре. Форма импульса $I(t)$ в период $t>0$ оставалась такой же, как на рис. 1. Результаты расчета представлены на рис. 3 .

Заметим, что качественные изменения величин переходных пиков $U_{m}$ и джоулевых потерь за импульс $W$ вполне ожидаемы. При этом высокая точность имитационного расчета тепловых потерь в каждом из сегментов позволяет обеспечить надежное согласование пробных значений $\delta T=T^{\prime}-T^{\prime \prime}$, задаваемых выше известными a priori, с искомыми значениями максимальной частоты генератора $f_{\max }$ и параметрами, характеризующими интенсивность внешнего охлаждения. Для этого сначала находится форма реального (плавного по $r$ ) распределения температуры $T(r)$ на основе решения стационарного уравнения теплопроводности $\left.\Delta_{r} T\right|_{1,2}=-\left\langle P_{1,2}\right\rangle / \kappa$ в слое $\mathbf{T}$. Здесь $\Delta_{r}-$ радиальная часть оператора Лапласа, $\kappa$ - теплопроводность материала теплоотвода. В правую часть введены ступенчатые распределения плотности мощности генерируемого тепла: $\left\langle P_{1}\right\rangle=f_{\max } W^{\prime} /\left(A^{\prime} h\right)$ для $0<r<r^{\prime}$ и $\left\langle P_{2}\right\rangle=f_{\max } W^{\prime \prime} /\left(A^{\prime \prime} h\right)$ для $r^{\prime}<r<r_{m}$, рассчитанные по значениям $W^{\prime}$ и $W^{\prime \prime}$ из предыдущей задачи. На границе между $\mathbf{T}^{\prime}$ и $\mathbf{T}^{\prime \prime}$ соблюдена непрерывность по $T$ и $d T / d r$, а в центре и на внешней границе сборки заданы условия $d T /\left.d r\right|_{r=0}=0$ и $\left.d T\right|_{r=r_{m}}=T_{\min }$. Затем из точных решений этого уравнения извлекаются усредненные по площадям отдельных сегментов $\mathbf{S}^{\prime}$ и $\mathbf{S}^{\prime \prime}$ и зависящие от параметров $f_{\max }$ и $T_{\min }$ значения $T_{1}$ и $T_{2}$.

В произвольном случае величины $T_{1}$ и $T_{2}$ не совпадают с заданными a priori $T^{\prime}$ и $T^{\prime \prime}$. Чтобы окончательно установить взаимно однозначное соответствие между внешними показателями $f_{\max }$ и $T_{\min }$, используем сходящуюся итерационную процедуру, в которой пробные значения $T_{\min }$ (значит, и $T_{1}$ и $T_{2}$ ) и назначаемые параметры $f_{\max }$, $T^{\prime}$ и $T^{\prime \prime}$ пошагово корректируются до достижения приемлемого уровня минимизации разностей $T_{1}-T^{\prime}$ и $T_{2}-T^{\prime \prime}$.

Рассчитанная этим способом зависимость максимальной частоты высоковольтного генератора (кривая 3 на рис. 3) от перепада $\delta T$ указывает на возможность ее изменения на порядок в диапазоне $3-30 \mathrm{kHz}$ при таком усилении интенсивности внешнего охлаждения, чтобы можно было обеспечить увеличение поперечного перепада температуры от 10 до $50 \mathrm{~K}$. Необходимая для этого мощность теплового потока во внешний радиатор, отнесенная к единичному звену, оценивается как $\Phi_{\text {out }}=\Phi^{\prime \prime}=f_{\max }\left(W^{\prime}+W^{\prime \prime}\right)$ и лежит в диапазоне 90-750 W.

Описанная методика расчета быстродействующих конструкций РВД в условиях с поперечным перепадом температуры вместе с оценками допустимых частот высоковольтных генераторов на их основе может послужить эскизной схемой для построения полной базы данных, 
необходимых при разработке новых модификаций РВД. Расширенное изложение развитого подхода планируется представить в отдельной публикации.

Работа выполнена при поддержке РНФ (проект № 14-29-00094).

\section{Список литературы}

[1] Grekhov I.V., Gorbatyuk A.V., Kostina L.S., Korotkov S.V., Jakovtchuk N.S. // Solid-State Electron. 1983. V. 26. N 11. P. 1132-1132.

[2] Schneider S., Podlesak T.F. // IEEE Trans. Plasma Sci. 2000. V. 28. N 5. P. 1520-1523.

[3] Gorbatyuk A.V., Grekhov I.V., Nalivkin A.V. // Solid-State Electron. 1988. V. 31. N 10. P. 1483-1491.

[4] Коротков С.В. // Приборы и техника эксперимента. 2002. № 4. C. 5-139.

[5] Wang H., He X., Chen W., Xie L., Zhou J., Xue B., Guo F., Zhang G., Chen Z., Zeng Z. // IEEE Trans. Power Electron. 2014. V. 29. N 4. P. 1553-1556. DOI: // 10.1109/TPEL.2013/2279072

[6] Коротков С.В., Жиодиков А.Л. // Приборы и техника эксперимента. 2011. № 1. С. 68-71.

[7] Коротков С.В., Аристов Ю.В., Коротков Д.А. // Приборы и техника эксперимента. 2012. № 3. С. 94-99.

[8] Горбатюк А.В., Иванов Б.В. // ЖТФ. 2015. Т. 85. В. 8. C. $94-99$.

[9] Горбатюк А.В., Иванов Б.В. // ФТП. 2017. Т. 51. В. 6. C. $835-843$.

[10] Грехов И.В., Месяи Г.А. // УФН. 2005. Т. 175. В. 7. С. $736-$ 1744.

[11] Synopsys Dev. Simulation. TCAD Sentaurus. http:www. synopsys.com 